\title{
HARDWARE IMPLEMENTATION OF TIME-SPATIAL FRAMING METHOD
}

\author{
PISZCZEK, M[arek]; RUTYNA, K[rzysztof]; KOWALSKI, M[arcin]; \\ SZUSTAKOWSKI, M[ieczyslaw] \& LUDWIKOWSKI, K[rzysztof]
}

\begin{abstract}
Laser Photography Device (LPD) is an innovative solution for precise imaging of defined fragment of space. Such systems can be used to recognize and identify terrorist threats as a part of public places monitoring system. An idea of this device is a registration of images (following image frames) by illuminating observed scene with very short laser impulses. In this article an idea, properties of method and constructional solutions are presented.

Keywords: range gated imaging, high speed photography systems, laser photography
\end{abstract}

\section{INTRODUCTION}

Modern vision and imaging systems offer big observation-measurement capabilities and these systems play huge role in present information systems. One of the potential applications of vision systems is security industry. The very important aspect of preventing terrorist threats is building an effective multispectral and multisensor system for open areas monitoring. Places like military bases, aerodromes, borders or sea ports $\tilde{n}$ places with big surface size are often being chosen to be a place for a terrorist attack.

One of very interesting group of imaging devices are ToF (time-of-flight camera) cameras. These cameras can be used in systems mentioned above. The ToF cameras are able not only to acquire an image but also to measure a distance from the detector to observed object. This property is the most characteristic for ToF cameras. ToF camera types can be divided into three groups according to the light source used and data acquisition method:

a) Impulse light source with digital time counters[1],

b) Modulated light source with phase detectors $[1,2]$, [1].

c) Impulse light source with gated acquisition time

Solution presented in this article is a device using time gating image acquisition method. The time-spatial framing method developed by authors is used to build Laser Photography Device (LPD). An active vision device for open space monitoring and terrorist threats detection is being built as an effect of recent work lead in the Institute of Optoelectronics, MUT (started in 2005). The LPD is destined to prevent and recognize possible terrorist threats in important land and marine areas.

\section{METHOD}

The ToF cameras are a major step in imaging devices development and because of their ability to acquire 3D information these cameras can replace recent stereoscopic systems to acquire spatial information. ToF cameras can be used to measure distances much more bigger than just single meters. Measurement ranges of ToF cameras can reach single kilometers thanks to using time-spatial framing method (with resolutions $\sim 0.5 \mathrm{~m}$ ).

Unique and typical properties of ToF cameras distinguish this particular type of imaging device. Functionalities of ToF cameras can be very useful and essential for many space information systems. Particular attention should be devoted to imaging information aspects connected with laser cameras:

a) An unique method of spatial information acquisition and visualization,

b) Selectivity of spatial imaging,

c) Hardware support for image quality enhancement,

d) Autosegmentation of observed scene that can be useful in hardware image processing,

e) Photogrammetric analysis of observed scene,

f) Scene and objects spatial modelling,

g) Autonavigation support for mobile platforms.

The essence of time-spatial framing method is the scene illumination and the detection type of image acquisition. The idea of a time-spatial framing method is presented in fig. 1. The key control parameters marked on Fig. $1-t_{O}, t_{M}, t_{D}$ are describing suitably illumination time, waiting time, detection time and $c$ - the speed of light. These parameters define:

a) Time interval of registered events $\Delta \mathrm{T}$,

b) The distance to observation area Rmin ,

c) The depth of observation area $\Delta \mathrm{R}$.

Acquisition of chosen time-spatial horizon (Fig.1) is achieved by selecting time sequences to control functional blocks of the Laser Photography Device.

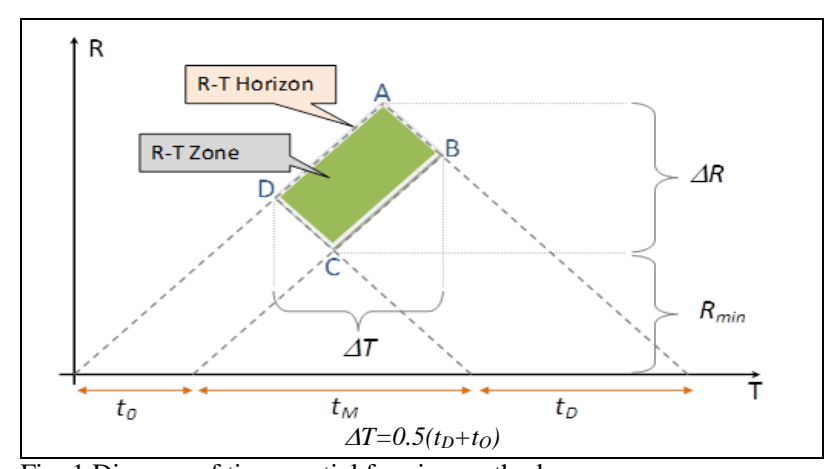

Fig. 1 Diagram of time-spatial framing method 

Laser photography is an active method. From a point of view of device work, natural light is a parasitic factor which should be eliminated. Thanks to using a laser source and proper spectral selection on a receiver module minimization of natural light impact can be achieved.

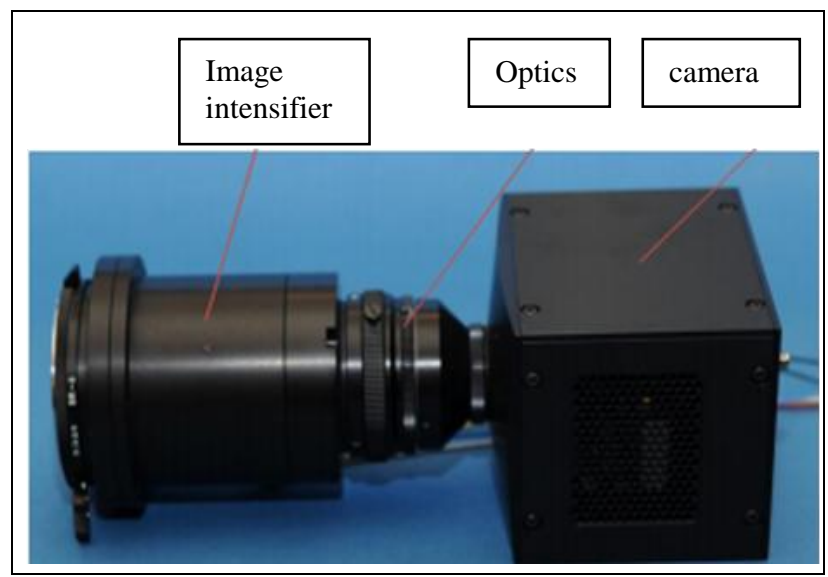

Fig. 6. A camera with image intensifier and wih optics

nThis means that image registration is independent of time of day - images acquired with daylight or during night are equal. Thanks to spatial image selection quality, enhancement can be achieved during image acquisition in adverse weather conditions e.g. fog. If the energy reaching the LPD is sufficient enough to synthesize an image then integrating a fragment of space will cause the contrast enhancement.

All of the information units are connected to the central iformation unit using an Ethernet interface. It is also possible to use wireless connection (e.g. WiFi) for data communication.

Because the main component of the Laser Photography System is the LPD, synchronization of every single information unit is achieved by a master system handling device.

\section{LPD HARDWARE IMPLEMENTATION}

Laser Photography Device is a reconfigurable device (Fig. 9). There are two types of the LPD system configuration:

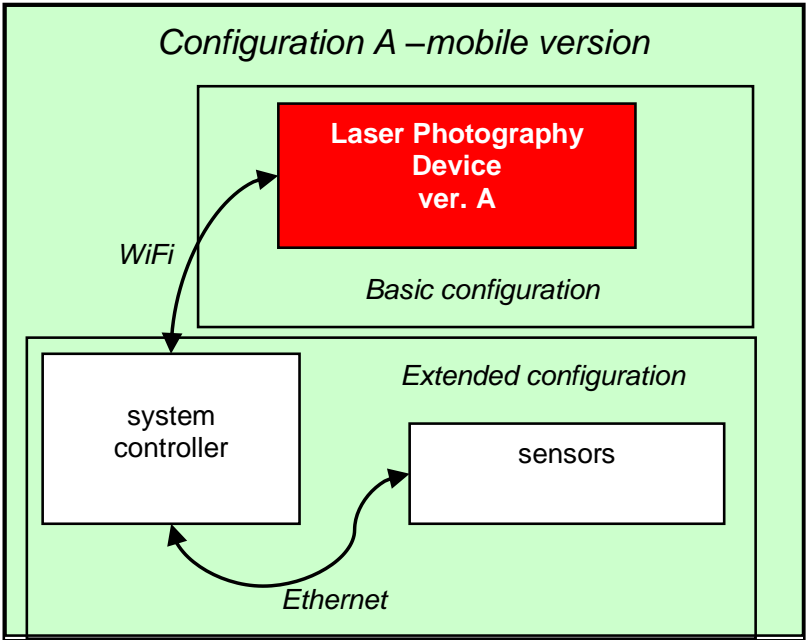

Fig. 7. Mobile version of the Laser Photography Device

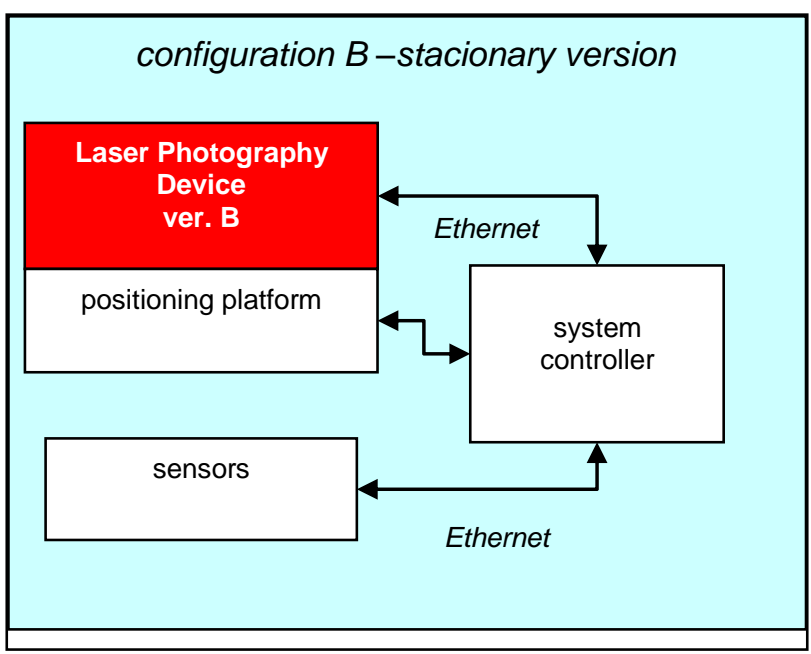

Fig. 8. Stacionary version of the Laser Photography Device

- ver. A ñ mobile configuration ñ equipped with solid state lasers. The device is handy and can be operated manually by a user. Images acquired by a mobile version of the LPD are presented directly on the LPDös display (Fig. 7).

- ver. B ก̃ stationary configuration $\tilde{\mathrm{n}}$ used with Nd:YAG laser. The whole device is placed on a positioning platform equipped with a set of sensors determining location and spatial orientation. Device control is realized by a central information unit (a system controller).

In a mobile version of the LPD all of the components (a camera, MCP image amplifier, controll module, solid state laser and computer unit) will be assembled in one housing (fig.6,8).

This causes that Laser Photography System (LPD with external sensors) will be compact and transportable. In addition the LPD will be equipped with a battery power supply. Mobile version of the LPD equipped with computer unit, TFT and touch displays will be used to browse registered images and to change image acquisition parameters without an external power source.

The second version of the Laser Photography Device is a unit equipped with an illuminator to acquire image data in long distance or in a difficult weather conditions. This version is a stationary version of the LPD (with high power Nd:YAG laser as an illuminator).

Another step in the LPD development will be an integration with external devices and sensors. The aim of the project is to build the Laser Photography System. The advantage of this system is open architecture as a result of using Ethernet protocol and interface.

Because research on the Laser Photography System is a work on a novel device and system following software solution is proposed:

a) research software - its task is to analyze the LPD work parameters and various system configurations,

b) systemös software $\tilde{\mathrm{n}}$ is the synthesis of different research solutions realizing specific system functions for various system configurations. 


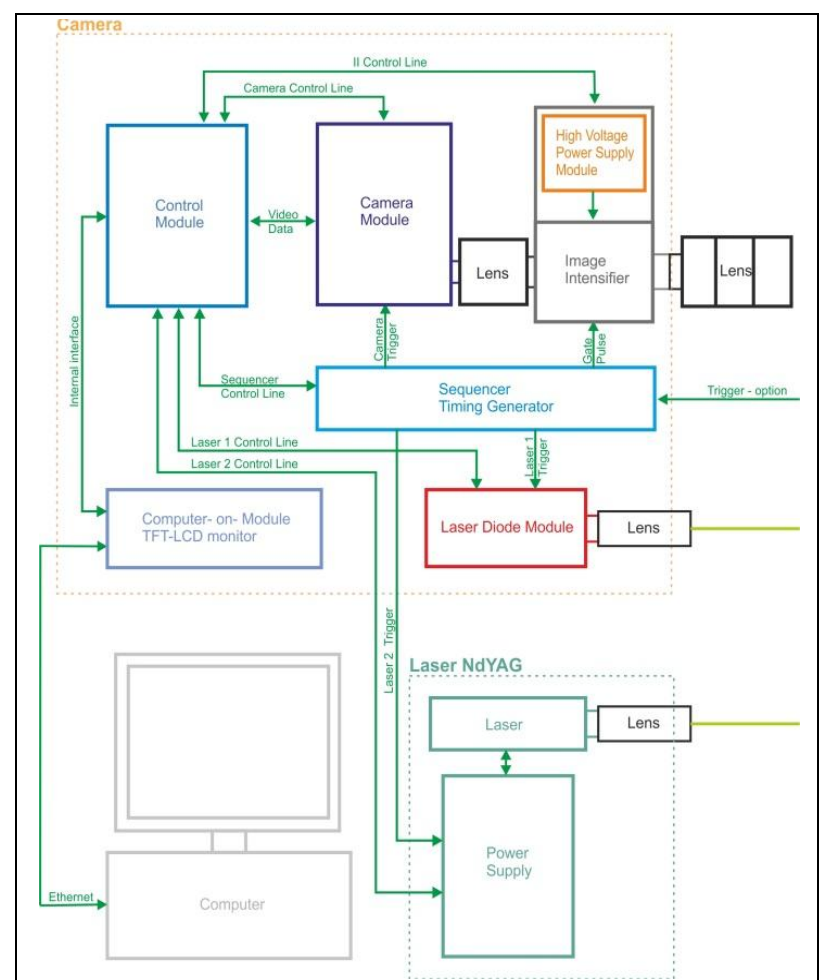

Fig. 9. Functional diagram of laser photography device

Classic imaging devices working in spectral range e.g. VIS-IR can register a space limited by an angular field of view. There is no such limitation in distance to observed object. Both VIS and IR cameras acquire information about direction to observed object as well as its angular size. Object detection by a VIS/IR camera can be a focusing factor for a system.

Application capabilities of such information can be strictly connected with metadata of every single information unit. Even if all cameras are spatially separated information about localization, spatial orientation and object observation direction can allow computation of observation coordinates for the LPD.

\section{CONCLUSIONS}

The Laser Photography Device is an innovative imaging device for large area security, defense and monitoring systems. Systems based on Laser Photography Devices can find applications not only in defense and security systems [5]. This device can be used by rescue services e.g. rescue workers searching for people. The device described in this article can be used to detect, visualize, recognize and identify unwanted actions of terrorists in strategic public places like airports, borders, industrial buildings (power plants, chemical plants) [4].

Selective space observation capability, one of ToF cameras property [7] allows to:

- three dimensional modelling and analysis of register events [8],

- long distance cars and ships registration tables reading [6],

- óthroughô windows imaging ñ ability to register images behind car or building windows,
- snipers detection (detection of gun optics) ñ identification of light reflections.

Further development of the Laser Photography Device in the area of improvement of camera parameters and adding new features seems to be justified. At this stage of development it can be said that using laser illumination can improve the process of defining acquisition parameters (time parameters as well as energetic).

All the analytical and construction work as well as many tests indicate that the potential offered by devices and systems using time-spatial framing method is immense. The Laser Photography Device which is a time-of-flight camera realizing the Range Gated Imaging method $[9,10]$ can become a valuable solution for spatial information and image recognition systems

\section{ACKNOWLEDGEMENTS}

This article was prepared based on development project realized in $12^{\text {th }}$ competition organized by Ministry of Science and Higher Education ñ óIntegrated Laser Photography System for open space monitoring and terrorist threats preventionô, OR00000312. Authors want to thank HARDsoft company for help and engagement in method and device development.

\section{REFERENCES}

[1] Schuon, S.; Theobalt, Ch.; Davis, J.; Thrun, S. (2008). Highquality scanning using time-of-flight depth superresolution. written at Anchorage, IEEE Computer Society Conference on Computer Vision and Pattern Recognition Workshops, 15th August, Alaska

[2] Medina, A (1992). Three Dimensional Camera and Rangefinder, January 1992. United States Patent 5081530

[3] Lellieviere S., Bonnier D. (2008), Introduction to Active imaging, Obzerv Technologies Inc., 400 Jean-Lesage, Suite 201, Qubec (Qubec), Canada, G1K 8W1

[4] Piszczek M., Rutyna K., Szustakowski M. (2007), Optoelectronic observation system with active illumination, International Congres on Optics and Optoelectronics 2007, SPIE Europe Optics and Optoelectronics Vol 65851L-1, 16-20th April 2007, Prague

[5] Piszczek M.,Rutyna K.,Szustakowski M. (2008), Imaging of space with using optoelectronic observation system with active illumination, European Physical Journal: Special Topics 154 (1), pp. 153-157

[6] Piszczek M., Rutyna K., Ludwikowski K. (2010), Aplikacyjne moŪiwoŞci fotografii laserowej (Laser photography application capabilities), Elektronika - Konstrukcje, Technologie, Zastosowania, 10/2010, Vol. 51, nr 10, pp. 36-39, ISSN: 08676747

[7] Lellieviere S., Bonnier D., Demers L.(2009), On the safe use of long-range laser active imager in the near-infrared, for Homeland Security, Obzerv Technologies Inc., 400, Jean-Lesage, Suite 201, Quebec, Qc, Canada G1K 8W1, Proceedings of SPIE Vol. 6406, Infrared Technology and Applications XXXII, pp. 1ñ9

[8] Stettner R.; Bailey H.; Richmond R.(2001); Eye-safe laser radar 3D imaging, Proceedings of SPIE 4377, 46 (2001)

[9] Andersson, P., "Long-range three-dimensional imaging using range-gated laser radar images", Opt. Eng. 45 034301, (2006)

[10] McDonald, T. E., Yates, G. J., Cverna, F. H., Gallegos, R. A., Jaramillo, S. A., Numkena, D. M., Payton, J. R., "Range-gated imaging experiments using gated intensifiersô, Proc. SPIE 3642, 142 (1999) 